\title{
Dislocation Core Phase Imaging by DBI
}

Silvia Penkova ${ }^{1,2}$ and Rodney A. Herring ${ }^{1 *}$

${ }^{1}$ CAMTEC, MENG, University of Victoria, British Columbia V8W 2 Y2 Canada.

${ }^{2}$ Babcock Canada Inc., Victoria, British Columbia V8Z 6R4 Canada.

* Corresponding author: rherring@uvic.ca

A method to image the phase existing at the core of a dislocation in a crystal, the smallest known physical singularity in existence, is demonstrated with remarkable clarity using diffracted beam interferometry (DBI) [1] enabling the measurement of the strain profile around the dislocation, at dislocation-dislocation intersections and the strain relief of the dislocation occurring at the free surface of the crystal. A dislocation in a single crystal Au specimen is used as an example. High contrast fringes within the interferogram were produced from the symmetrical interference of the $220 \mathrm{~g}$ and $-2-20 \mathrm{~g}$ beams, i.e., $\pm g h \mathrm{kl}$, interfered on the optic axis by means of a biprism (Fig. 1), a configuration that cancels the Cs aberrations of the two beams. In the perfect crystal, i.e., region without defects, the equal but opposite Bragg diffracted beams also cancels each other's phase. The remaining phase of the dislocation core carried by the two interfering Bragg diffracted beams captures the strain around the dislocation core by measuring its phase shift. This is possible as each Bragg diffracted beam diffracts off a different atomic plane situated on either side (left side (LS) + right side, (RS) of the dislocation core (Fig 2a) so its phase is not cancelled. The phase contributions in the DBI interferogram can be expressed as,

$$
\begin{aligned}
& \Delta \phi=\phi_{g_{h k l}}+\phi_{d i s l_{-}{ }{ } o r e_{\mathbf{L S}}}+\phi_{g_{\bar{h} \bar{k} \bar{l}}}+\phi_{\text {disl_core }_{\mathbf{R S}}}
\end{aligned}
$$

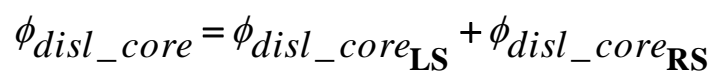

$$
\begin{aligned}
& \Delta \phi=\phi_{\text {disl_core }}
\end{aligned}
$$

Each Bragg diffracted beam carriers half of the phase of the dislocation core $\left(\phi_{\text {disl_c }_{\text {core }}} \mathbf{L S}+\phi_{\text {disl_core }}{ }_{\mathbf{R S}}\right)$. Together when the two beams are directly overlapped by DBI they combine to produce the whole phase shift of the dislocation core $\left(\phi_{d i s} l_{-}\right.$core $)$. In this case, the phase shift is due to the strain of the displaced atomic planes. Other phase shifts included an intersection point with two other dislocations as well as the strain relief of the dislocation at the top and bottom surfaces of the crystal (Fig. 3). Strain from a nearby stacking fault produced the slope in the phase profile along the length of the dislocation seen in Fig. 3d.

This new phase imaging method of dislocations will help better understand the strain profile around their core, their intersection points with other dislocations and at the free surfaces. It may be possible to also image the phase shifts at dislocations decorated with precipitates, alloying additions and impurities and possibly the electrostatic charges due to unbonded electrons and holes existing at the dislocation cores of semiconductors and ceramics.

Dislocations are arguably the most important defect in materials having a strong influence on their physical, electrical, photonic, sensory and magnetic properties as well as on the rate and morphology of corrosion. Their presence in the active region of electronic devices causes their failure.

Grants from UVic, NSERC Discovery, Engage and CRD, CFI and BCKDF are appreciated. 
References:

[1] Herring, R.A., G. Pozzi, and T. Tanji J. of Electron Microscopy 424 (1993) 267.

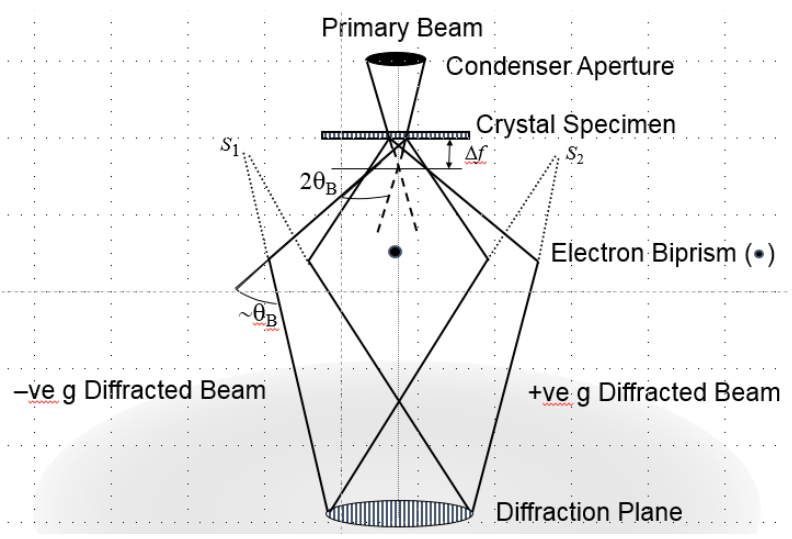

Figure 1. Configuration of DBI using the symmetric interference on axis of the $220 \mathrm{~g}$ and $-2-20 \mathrm{~g}$ beams of $\mathrm{Au}$ by means of the electron biprism to image dislocations in the crystal specimen.
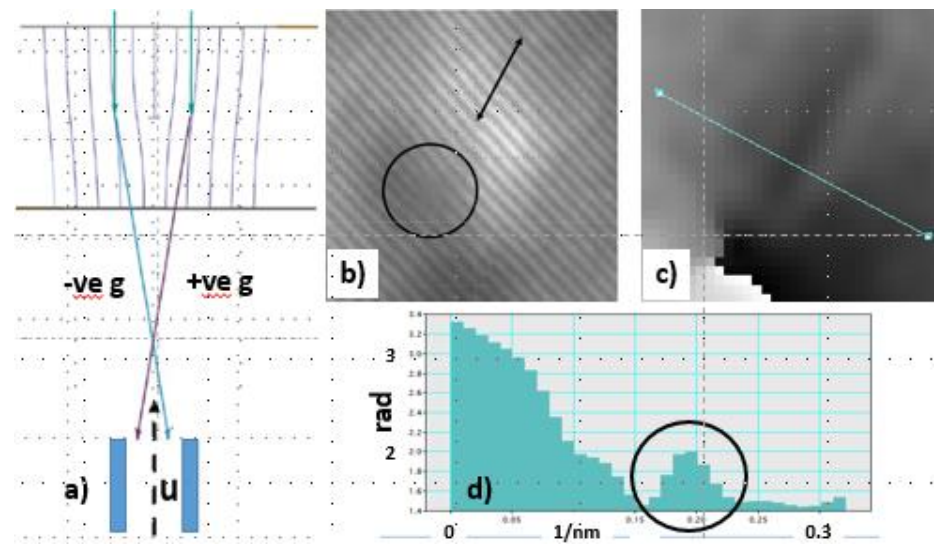

Figure 2. a) Displaced intensity contrast (blue bars) of Bragg diffracted beams occurs to one side of the dislocation core indicated by its line direction, u. b) DBI of a dislocation running along the black arrowed line with its $2 \pi$ phase shift shown (circle), c) its reconstructed phase image reveals the dislocation and along the blue arrow is $d$ ) the phase profile of the dislocation core (circled).
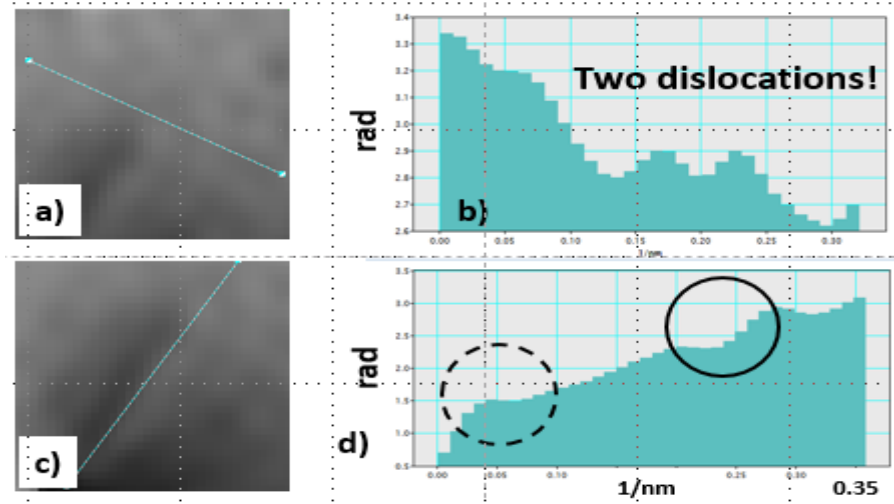

Figure 3. a) and b) the phase shift and profile of two dislocations intersecting, c) and d) the phase shift and profile along the length of the dislocation revealing the intersection point of the two dislocations (solid circle) as well as the relief of the dislocation's strain at one of the crystal's surfaces (dashed circle). 